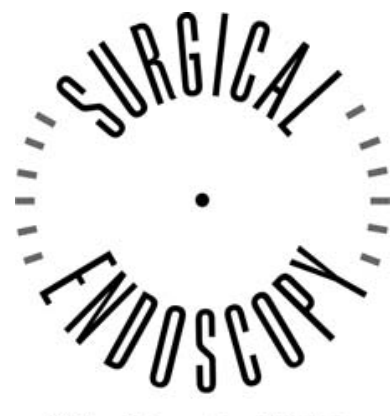

and Other Interventional Techniques

\title{
Thoracoscopic tracheoaortopexia for the treatment of life-threatening events in tracheomalacia
}

\author{
D. C. van der Zee, N. M. A. Bax \\ Department of Pediatric Surgery, Wilhelmina Children's Hospital, KE 04.140.5, P.O. Box 85090, 3508 AB Utrecht, The Netherlands
}

Received: 27 September 2006/Accepted: 25 November 2006/Online publication: 14 March 2007

\begin{abstract}
Background: Life-threatening events resulting from tracheomalacia are a well-known complication of infants with esophageal atresia. Aortopexy is accepted as the most effective method for managing severe life-threatening and localized tracheomalacia with a success rate of $85 \%$ to $90 \%$. Since the advent of minimally invasive surgery (MIS), the procedure also can be performed using thoracoscopic MIS.

Methods: Between January 2002 and November 2005, six children with esophageal atresia were treated using MIS for life-threatening events attributable to tracheomalacia.

Results: The patients tolerated the thoracoscopic procedure well, and all tracheoaortopexies could be performed thoracoscopically. There were two recurrences, which could be treated using thoracoscopy. After a follow-up period of 27 months (range, 10-45 months), all the patients are doing well and have had no more lifethreatening events.

Conclusions: Although this is the largest thoracoscopic series to date, the series is too small for any conclusions yet to be drawn. Thoracoscopic tracheoaortopexia is feasible and offers the advantages of MIS.
\end{abstract}

Key words: Aortopexy - Children - Thoracoscopy - Tracheomalacia

Tracheomalacia is a generalized or localized weakness of the tracheal wall that can cause luminal obstructions at times of increased intrathoracic pressure such as expiration, crying, or coughing [7]. Life-threatening events attributable to tracheomalacia are a well-known complication for infants with esophageal atresia. Because the aortic arch and trachea are closely related, elevation of the aortic arch against the posterior surface of the sternum pulls the trachea up as well, thus alleviating the complaints from the collapse of the anterior and posterior walls of the trachea.

Aortopexy is accepted as the most effective treatment method for severe life-threatening and localized tracheomalacia, with a success rate of $85 \%$ to $90 \%[7,8]$. This procedure usually is performed by either a thoracotomy or a cervical approach [3]. Since the advent of minimally invasive surgery (MIS), the procedure also can be performed by thoracoscopic MIS [2].

\section{Materials and methods}

Between January 2002 and November 2005, six children (4 girls and 2 boys) with esophageal atresia were treated by MIS for life-threatening events attributable to tracheomalacia. The mean patient age at the time of surgery was 5 months (range, 14 days to 12 months), and the mean patient weight was $5.5 \mathrm{~kg}$ (range, $3-9 \mathrm{~kg}$ ).

The technique was described earlier by DeCou et al. [2]. In brief, the procedure is started with a tracheoscopy and bronchoscopy under spontaneous breathing anesthesia. "Kissing" of the ventral and posterior tracheal wall are an indication for thoracoscopic tracheoaortopexia.

The child is placed in a supine position with a tilt of the left shoulder to facilitate the approach of the anterior mediastinum from the left side. One 6-mm trocar for the camera and two $3.5-\mathrm{mm}$ trocars for instrumentation are used. Under identification of the phrenic nerve, the pleura can be opened and the left thymus lobe can be mobilized and pushed to the right side of the mediastinum or resected if necessary. The pericardium, the ascending aorta, and the brachiocephalic artery then can be identified and prepared where necessary to give adequate access for placement of the sutures. By pushing on the sternum, the best location for the sutures can be determined. Small stab wounds are made and 3-0 Ethibond (Cincinnati, $\mathrm{OH}$ ) nonabsorbable sutures can be introduced through the sternum, either by straightening of the needle somewhat and direct puncture or with the use of an Endoclose (TYCO Medical, Mansfield, MA). Three to four transsternal sutures can be placed from the pericardium up to the brachiocephalic artery. After the sutures have been placed and pulled against the posterior surface of the sternum, tracheobronchoscopy is repeated to determine the efficacy of the tracheoaortopexia. Where necessary, additional sutures can be placed. At the end of the procedure, the pneumothorax is evacuated under direct vision.

The trocar holes and stab wounds are closed with either absorbable Vicryl $4-0$ or 5- 0 sutures. No drainage is left in place. The chil- 
dren are kept in the intensive care unit overnight only if the anesthesiologist deems it necessary.

\section{Results}

The patients tolerated the thoracoscopic procedure well, and all tracheoaortopexies could be carried out thoracoscopically. Tracheoscopy at the end of the procedure demonstrated a clear improvement in the tracheal diameter.

Two patients experienced recurrence of life-threatening events after 2 and 4 weeks, respectively. For the first patient, two additional sutures were placed on the arteria bracheocephalica. Reexploration of second patient found only one suture. The other sutures seem to have dissolved and could not be retrieved. Three new sutures were placed. After a follow-up period of 27 months (range, 10-45 months), all the patients were doing well and had no more life-threatening events.

\section{Discussion}

Life-threatening events for children with esophageal atresia may occur because of gastroesophageal reflux or tracheomalacia [3]. Tracheomalacia may be caused by a malformation of the tracheal rings and can occur to a more or lesser extent among children born with esophageal atresia that has a distal fistula [3]. The occurrence of a life-threatening event is an indication for surgery.

In the past, an antireflux procedure would be the first step in the treatment of a life-threatening event [3]. Currently, with a more accurate diagnosis of tracheomalacia by tracheobronchoscopy, there is a greater tendency to perform the aortopexy as a first step [4]. The approach may be by a left or right thoracotomy or by a cervical incision [3]. Because the adjacent aorta and trachea are closely related, elevation of the aorta against the posterior surface of the sternum also lifts the anterior wall of the trachea, thus alleviating the life-threatening dyspnea. Reported series, however, are small [1, 8], and a recent Cochrane Review showed no randomized controlled trials at all [5].

More recently, case histories of a thoracoscopic approach also have been described [2,6]. The patients seemed to benefit from the procedure, experiencing adequate relief from the life-threatening events, and the children recovered rapidly. The success rate is described to be $85 \%$ to $90 \%[7,8]$.

This report describes the thoracoscopic tracheoaortopexia experienced by six children seen during an interval of nearly 4 years. For two of these children, a thoracoscopic redo procedure was necessary due to recurrence of life-threatening events after 2 and 4 weeks, respectively.

In the first case, the child probably had not yet been breathing spontaneously enough for the true effect of the procedure to be determined. Otherwise, it would have been noted that the procedure was not performed sufficiently upward toward the brachiocephalic artery to alleviate the symptoms. The lesson learned from this case is that patients must truly be breathing spontaneously for the adequacy of the effect to be determined.

In the second case, only one suture could be retrieved. It is always a question of finding the subtle balance between a suture being placed too superficially, as in this case, resulting in recurrence, and one being placed too deep, resulting in blood obscuring the vision. The latter is more bothersome in MIS than it would be in open surgery. Because haptic feedback is more difficult with MIS, this may be a matter of a learning curve.

All the children tolerated the procedure well, and besides the recurrence, no other adverse effects occurred. Even the children who underwent a redo procedure demonstrated no adverse effects. Moreover, one operation report for the redo procedure described the location as one not managed with surgery previously.

Although this is the largest series to date, it is too small for any conclusions yet to be drawn. Thoracoscopic tracheoaortopexia is feasible and offers the advantages of MIS. The two recurrences indicate that there is a learning curve. Recently, a retrospective multicenter study on thoracoscopic repair of esophageal atresia experienced by 104 children was published [4]. If it would be possible to set up a multicenter prospective study on thoracoscopic tracheoaortopexia, a larger series could be gathered to describe a more definite outcome for MIS treatment of tracheomalacia.

\section{References}

1. Abdel-Rahman U, Ahrens P, Fieguth HG, Kitz R, Heller K, Moritz A (2002) Surgical treatment of tracheomalacia by bronchoscopic monitored aortopexy in infants and children. Ann Thorac Surg 74: 315-319

2. DeCou JM, Parson DS, Gauderer MWL (2001) Thoracoscopic aortopexy for severe tracheomalacia. Pediatr Endosurg Innovative Tech 5: 205-208

3. Harmon CM, Coran AG (1998) Ch 62. Congenital anomalies of the esophagus. In: O’Neill JA Jr, Rowe MI, Grosfeld J, Fonkalsrud EW, Coran AG (eds) Pediatric surgery, 5th ed. Mosby Year book, New York, pp 941-965

4. Holcombe GW III, Rothenberg SS, Bax NMA, Martinez-Ferro M, Albanese CT, Ostlie DJ, van der Zee DC, Yeung KC (2005) Thoracoscopic repair of esophageal atresia and tracheoesophageal fistula. Ann Surg 3: 422-428

5. Masters I, Chang A, Masters IB (2005) Intervention for primary (intrinsic) tracheomalacia in children. Cochrane Database Syst Rev 19: CD005304

6. Schaarschmidt K, Kolberg-Schwerdt A, Pietsch L, Bunke K (2002) Thoracoscopic aortopericardiosternopexy for severe tracheomalacia in toddlers. J Pediatr Surg 37: 1476-1478

7. Sumit D, Currie BG (2006) The role of aortopexy in severe tracheomalacia. J Pediatr Surg 41: 533-537

8. Weber TR, Keller MS, Fior A (2002) Aortic suspension (aortopexy) for severe tracheomalacia in infants and children. Am J Surg 184: 573-577 